\title{
A Prototype MATLAB Code Critiquer
}

\author{
Marissa Walther \\ mlwalthe@mtu.edu \\ Michigan Technological University \\ Houghton, MI, USA
}

\author{
Leo C. Ureel II \\ ureel@mtu.edu \\ Michigan Technological University \\ Houghton, MI, USA
}

\author{
Charles Wallace \\ wallace@mtu.edu \\ Michigan Technological University \\ Houghton, MI, USA
}

\begin{abstract}
This poster presents MATLAB Critiquer, a system that examines code submissions and provides feedback to first year engineering students who are learning to program in MATLAB. Code Critiquers act as substitute mentors, when instructors are not available, and give students experience with interactive, test-driven development. [5] Students using our critiquer are engaged in communication by proxy with the instructor. This communication does not replace instructor feedback; rather, it codifies common feedback scenarios to assist the instructor in reaching students just when the student is engaged in problem solving and learning.

Our critiquer uses the MATLAB Java API; connecting to the MATLAB Engine that parses and executes student code submissions. [4] We process error messages that are returned using regular expressions, then use these results to craft feedback for the students. Static code analysis is used to provide feedback on style. The critique covers code structure, shakedown test results, and programming style in a manner appropriate for novice coders.
\end{abstract}

\section{CCS CONCEPTS}

- Social and professional topics $\rightarrow$ Computer science education; Computer engineering education; Student assessment; • Applied computing $\rightarrow$ Interactive learning environments; • Software and its engineering $\rightarrow$ Agile software development.

\section{KEYWORDS}

Code Critiquer, Autograder, MATLAB, Engineering Fundamentals

ACM Reference Format:

Marissa Walther, Leo C. Ureel II, and Charles Wallace. 2019. A Prototype MATLAB Code Critiquer. In Innovation and Technology in Computer Science Education (ITiCSE '19), July 15-17, 2019, Aberdeen, Scotland Uk. ACM, New York, NY, USA, 1 page. https://doi.org/10.1145/3304221.3325591

\section{SIGNIFICANCE OF THE TOPIC}

Increasingly, students across campus are required to learn some form of programming. Students with little or no programming experience can be easily confused or intimidated, so the feedback they get (both human and automated) must be carefully designed and targeted to novices. We believe that professional grade tools (e.g., development environments, style checkers, unit test frameworks),

Permission to make digital or hard copies of part or all of this work for personal or classroom use is granted without fee provided that copies are not made or distributed for profit or commercial advantage and that copies bear this notice and the full citation on the first page. Copyrights for third-party components of this work must be honored. For all other uses, contact the owner/author(s).

ITiCSE '19, fuly 15-17, 2019, Aberdeen, Scotland Uk

(c) 2019 Copyright held by the owner/author(s).

ACM ISBN 978-1-4503-6301-3/19/07.

https://doi.org/10.1145/3304221.3325591 designed for experts, provide feedback that is inappropriate and even counterproductive for novices.

Furthermore, not only are non-CS majors learning to program, but many non-CS faculty find themselves teaching programming courses and relying on professional tools to help provide feedback to their students. Besides large-scale initiatives in High Performance Computing, Big Data, and basic Computer Science Research, more and more computation-centric degrees are being offered by universities. Many students in non-computing majors must take programming courses to develop the required computational background for their field. For instance, the MATLAB language for numerical computing is commonly introduced to engineering students of all disciplines.

Through an automated MATLAB Critiquer, we can give engineering students early experience with interactive, test-driven software development. This exposure will prepare them better for today's computer-centric workplace. It will also reduce the frustration that students often experience in early programming projects, mitigating the risk of student burnout and helping with retention in computing-related majors.

\section{CONTENT}

In this poster we present a code critique for MATLAB. We provide examples of MATLAB code submitted by students and the feedback reported by our MATLAB Critiquer. We discuss the different kinds of feedback provided to students: code structure, shakedown test results, and programming style. A one-page handout will be available that describes our critiquer.

\section{REFERENCES}

[1] Stephen H. Edwards and Manuel A. Perez-Quinones. 2008. Web-CAT: Automatically grading programming assignments. Proceedings of the 13th annual conference on Innovation and technology in computer science education - ITiCSE '08 (2008).

[2] Marios Fokaefs, Nikolaos Tsantalis, Eleni Stroulia, and Alexander Chatzigeorgiou. 2011. JDeodorant: Identification and Application of Extract Class Refactorings. Proceeding of the 33rd international conference on Software engineering - ICSE ' 11 (2011).

[3] Mike Joy, Nathan Griffiths, and Russell Boyatt. 2005. The boss online submission and assessment system. Fournal on Educational Resources in Computing (FERIC) 5 , 3 (2005), 2.

[4] Inc. Mathworks. [n.d.]. MATLAB. https://www.mathworks.com/products/matlab. html

[5] Lin Qiu and Christopher Riesbeck. 2008. An incremental model for developing educational critiquing systems: experiences with the Java Critiquer. Fournal of Interactive Learning Research 19, 1 (2008), 119-145.

[6] Jaime Spacco, David Hovemeyer, William Pugh, Fawzi Emad, Jeffrey K. Hollingsworth, and Nelson Padua-Perez. 2006. Experiences with marmoset: designing and using an advanced submission and testing system for programming courses. Proceedings of the 11th annual SIGCSE conference on Innovation and technology in computer science education - ITICSE '06 (2006).

[7] Arto Vihavainen, Matti Luukkainen, and Martin Pärtel. 2013. Test my code: An automatic assessment service for the extreme apprenticeship method. In 2nd International Workshop on Evidence-based Technology Enhanced Learning. Springer, 109-116. 